\title{
Numerical simulation of microstructure formation during solidification and heat treatments of Ni-base superalloys
}

\author{
L. Rougier ${ }^{1,3}$, A. Jacot ${ }^{1}$, Ch.-A. Gandin ${ }^{2}$, P. Di Napoli ${ }^{1}$, P.-Y. Théry ${ }^{3}$, V. Jaquet ${ }^{3}$ \\ ${ }^{1}$ LSMX, MXG, Ecole polytechnique Fédérale de Lausanne, 1015 Lausanne, Switzerland \\ ${ }^{2}$ CEMEF UMR CNRS 7635, MINES ParisTech, 06904 Sophia Antipolis, France \\ ${ }^{3}$ Snecma-SAFRAN group, Service YQGC, 92702 Colombes, France
}

Keywords: Numerical modelling, precipitation, heat treatment, microsegregation

\begin{abstract}
A comprehensive modelling approach has been developed for the simulation of microstructure formation during solidification and heat treatment in Ni-base superalloys. The microsegregation taking place during solidification is simulated using the pseudofront tracking (PFT) technique. This finite volume method calculates the concentration profiles in primary $\gamma$ and the proportion of liquid and $\gamma^{\prime}$ in the interdendritic regions. The same model is then used to describe the dissolution of the interdendritic $\gamma$ ' particles during the subsequent solution heat treatment. The concentrations in primary $\gamma$ predicted with the PFT model are then used to calculate the evolution of the $\gamma^{\prime}$ precipitates and their size distributions at different locations in a dendrite arm during heat treatment. This is achieved with a precipitate size distribution (PSD) model. The classes of the distribution are created and tracked based on classical nucleation theory and a semi-analytical model for the growth of the precipitates as a function of their radius and the matrix supersaturation. The PFT and PSD models are both coupled with Thermo-Calc, which is used for the computation of the concentrations at the $\gamma /$ liquid and $\gamma / \gamma$, interfaces, for the Gibbs-Thomson effect in precipitates, and to calculate non-diagonal diffusion matrices based on a mobility database. The precipitation model was applied to a $\mathrm{Ni}-\mathrm{Al}-\mathrm{Cr}$ alloy and the results were compared with experimental data from the literature. Experimental and simulation results are in good qualitative agreement. The main phenomena taking place during precipitation, i.e. nucleation, growth and coarsening, are well reproduced by the precipitation model. Some discrepancies were observed on the precipitate number density, which were attributed to the homogeneous nucleation model. The full modelling sequence was then applied to $\mathrm{Ni}-\mathrm{Al}$ in order to assess the influence of an incomplete homogenization heat treatment on the formation of $\gamma^{\prime}$ precipitates.
\end{abstract}

\section{Introduction}

Nickel based superalloys are commonly used for aeronautical applications for their outstanding mechanical properties and oxidation resistance at high temperatures [1]. The mechanical properties of these alloys are obtained by structural hardening, which is associated with a high volume fraction of $\gamma^{\prime}$ precipitates in a $\gamma$ matrix. Over the last two decades a considerable effort has been made to develop numerical models for precipitation [2-11]. Most of the models are based on the theory of Langer and Schwarz [2] or the modified form of Kampman and Wagner [3]. In the latter approach, nucleation, growth and coarsening phenomena are addressed in a physical manner and the particle size distribution is directly represented. Specific examples are found in refs. [4-7].
Most of the precipitation models proposed in the literature address precipitation without considering the influence of solidification. Decoupling the different processing steps may be appropriate since many phenomena do not occur simultaneously, or have only limited reciprocal influence. For example, the solidification microstructure may be totally erased during a solution heat treatment before ageing, which makes it unnecessary to consider solidification and heat treatment within the same approach. In such a case, precipitation can be described simply by considering a matrix of nominal composition as an initial condition. However, it is very common that inhomogeneities in the solidification microstructure survive the solution heat treatment, which introduces local variations of the precipitation response. For this reason, integrated approaches have been developed to address solidification, homogenization and precipitation within a same approach [8-11].

So far integrated approaches were essentially applied to precipitation in $\mathrm{Al}$ alloys. The present work is an attempt to extend it to Ni-base superalloys, which present additional difficulties due to the important role of the non-diagonal terms in diffusion matrices and the high volume fractions of precipitates.

\section{Model description}

The present work is based on the approach of Gandin and Jacot [11], which was originally used to investigate the influence of solidification conditions on the precipitation and the formation of a precipitate-free zone (PFZ) during the treatment of a continuously cast AA3003 alloy. Two models are combined. First, a Pseudo Front-Tracking (PFT) method [12] is used to calculate the solute concentration profiles at the scale of the secondary dendrite arm spacing and to describe the formation/dissolution of interdendritic phases. The second model is a Particle Size Distribution (PSD) model used to simulate the precipitation in a given location of the primary phase. It can be applied to several volume elements of the PFT model as illustrated in Figure 1. These models were modified in order to take into account crossdiffusion, which is an important phenomenon in Ni-base superalloys, due to the high fraction of solute elements.

\section{Solidification/homogenization model}

The spatial distribution of the solute in primary $\gamma$ during solidification and homogenization is calculated using the PFT model of Jacot and Rappaz [12]. The model is applied to a 1D domain with a Cartesian geometry, whose size is defined by the Dendrite Arm Spacing (DAS), as shown in Figure 1. The model is based on a finite volume method and a fixed mesh. The diffusion equation is numerically solved for each volume element of the domain: 


$$
\frac{\partial c_{i}^{v}}{\partial t}=-\nabla \cdot\left(J_{i}^{v}\right)
$$

where $v(=l, \gamma)$ is a phase superscript and $i$ is a solute index. The flux $J_{i}^{v}$ is computed from the concentrations gradients:

$J_{i}^{v}=-\sum_{j} D_{i j}^{v} \nabla c_{j}^{v}$

where $D_{i j}^{V}$ denotes the terms of the diffusion matrix.

During solidification the $\gamma /$ liquid interface is positioned in a given (mixed) volume element in which a solute balance is formulated Assuming local equilibrium, the interface concentrations in $\gamma$ and $l$ are obtained from Thermo-Calc based on the average composition in the mixed volume element. The displacement of the interface is handled through state transitions of the finite volume elements, from $l$ to $\gamma / l$ and $\gamma$. Further details of the method can be found in [12].

When some undercooling for the nucleation of $\gamma^{\prime}$ in the remaining liquid is reached, interdendritic $\gamma^{\prime}$ formation is predicted using the method described by Du and Jacot [13]. In this approach the interdendritic region is considered as a uniform mixture of liquid and solid phases at thermodynamic equilibrium. The model is based on the back-diffusion fluxes at the primary $\gamma /$ mixture interface, and solute balances are performed over the interdendritic domain for all solute elements. The model incorporates a secondary phase distribution parameter allowing for the distinction between coupled and divorced eutectics [13]. The calculations are coupled with the phase diagram software Thermo-Calc to obtain the interface concentrations in primary $\gamma$ and to compute thermodynamic equilibrium in the mixture, i.e. to continuously update the volume fractions and concentrations of the interdendritic phases according to the new temperature and average concentrations in the interdendritic region.

After the liquid has disappeared from the interdendritic phase mixture, the calculation is continued so that evolution of the interdendritic $\gamma^{\prime}$ during the solution/homogenization heat treatment can be predicted. The dissolution of $\gamma^{\prime}$ is controlled by the back diffusion in primary $\gamma$.

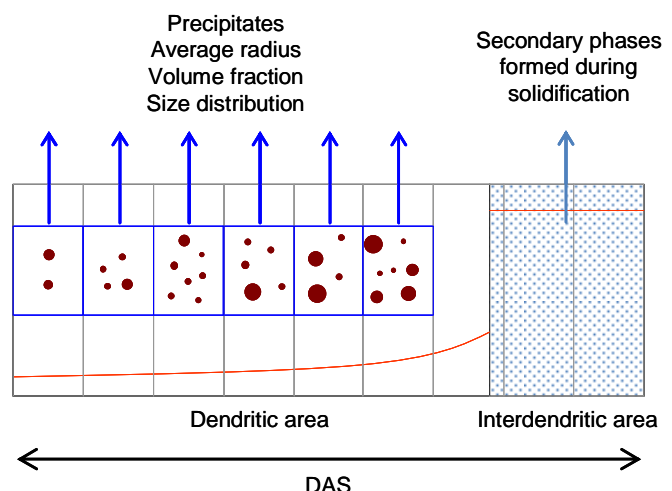

Figure 1 : Schematic representation of precipitation calculations performed in several volume elements of the dendritic domain used to simulate microsegregation induced by solidification.

\section{Precipitation model}

Precipitation is simulated using a model based on the discretization of the Particle Size Distribution (PSD) into size classes [3]. Each class corresponds to a category of precipitates, having its own values for the radius, $R_{(k)}$, the particle number density, $n_{(k)}$, and the interface concentrations. The classes are created during the nucleation stage. At each time $t[\mathrm{~s}]$ and temperature $T[\mathrm{~K}]$, the nucleation rate is calculated according to classical nucleation theory $[14,15]$ :

$$
I=N_{0}\left(1-g^{\gamma^{\prime}}\right) \mathrm{Z} \beta e^{-\frac{\Delta G^{*}}{k_{B} T}} e^{-\frac{\tau}{t}}
$$

where $N_{0}$ is the potential density of nucleation sites per unit volume $\left[\mathrm{m}^{-3}\right], g^{\gamma}$ is the $\gamma^{\prime}$ volume fraction introduced in order to take into account the decrease of potential nucleation sites density, $Z$ is the Zeldovich factor, $\beta$ is the condensation rate of the solute atoms on a stable precipitate $\left[\mathrm{s}^{-1}\right], \tau$ is the incubation time [s] and $k_{B}$ is the Boltzmann constant [J. $\left.\mathrm{K}^{-1} \cdot \mathrm{mol}^{-1}\right] . \Delta G^{*}$ is the critical energy for homogeneous nucleation $[\mathrm{J}]$ and, for a spherical nucleus, it is given by:

$\Delta G^{*}=\frac{16 \pi}{3} \frac{\left(\sigma^{\gamma / \gamma^{\prime}}\right)^{3}}{\left(\Delta G_{V}\right)^{2}}$

where $\sigma^{\not \gamma \gamma}$ is the interfacial energy [J.m $\left.{ }^{-2}\right] . \Delta G_{V}$ is the driving force for nucleation per unit volume $\left[\mathrm{J}^{-3}\right]$, calculated by Thermo-Calc from the average concentrations. The time-step is adapted in order to obtain an approximately constant number of precipitates in each new class.

A Lagrangian approach $[16,17]$ is then used to compute the evolution of the radius of each precipitate class. The growth rate in a class $k$ of radius $R_{(k)}$ is calculated using the solute fluxes in the matrix at the interface and the effective diffusion matrix:

$v_{(k)}\left(c_{i(k)}^{\gamma^{* *}}-c_{i(k)}^{\gamma^{*}}\right)=\left.\sum_{j} D_{i j}^{\gamma} \frac{\partial c_{j(k)}^{\gamma}}{\partial r}\right|_{r=R_{(k)}}$

where $c_{i(k)}^{\gamma *}$ and $c_{i(k)}^{\gamma^{*}}$ are the interface concentrations in solute element $i$ in class $k$ in the $\gamma$ and $\gamma^{\prime}$ phases, respectively. The main problem consists in the calculation of a unique growth rate satisfying simultaneously eq. (5) for all solute elements and the conditions given by local thermodynamic equilibrium. First, some assumptions have to be made for the concentration profile in the matrix. Assuming a stationary concentration profile around a spherical precipitate, the following analytical solution can be used $[7,18]$ :

$\left.\frac{\partial c_{i(k)}^{\gamma}}{\partial r}\right|_{r=R_{(k)}}=\frac{\bar{c}_{i(k)}^{\gamma}-c_{i(k)}^{\gamma^{*}}}{\delta_{i(k)}}$

with

$\delta_{i(k)}=\frac{\Omega_{i(k)}}{2 \lambda_{i(k)}^{2}} R_{(k)}$ 
and

$$
2 \lambda_{i(k)}^{2}-2 \lambda_{i(k)}^{3} \sqrt{\pi} \exp \left(\lambda_{i(k)}^{2}\right) \operatorname{erfc}\left(\lambda_{i(k)}\right)=\Omega_{i(k)}
$$

where $\bar{c}_{i(k)}^{\gamma}$ is the average matrix concentration, $\delta_{i(k)}$ is the characteristic diffusion length, and $\Omega_{i(k)}=\left(\bar{c}_{i(k)}^{\gamma}-c_{i(k)}^{\gamma^{*}}\right) /\left(c_{i(k)}^{\gamma^{* *}}-c_{i(k)}^{\gamma^{*}}\right)$ is the supersaturation.

The system of eqs (5)-(8) is solved for each size class k. A small volume is defined in the vicinity of the $\gamma / \gamma^{\prime}$ interface. An equilibrium calculation is then performed in this domain in order to obtain the new interface concentrations and the volume fraction variation, which is translated into a growth rate. The GibbsThomson effect is taken into account by adding the corresponding contribution to the Gibbs free energy to the $\gamma$ ' phase.

Finally, the new radius of the precipitate class is obtained from the growth rate:

$R_{(k)}^{t+\Delta t}=R_{(k)}^{t}+v_{(k)} \cdot \Delta t$

where $\Delta t$ is the time step.

Average quantities, such as the total precipitates density $(N)$, the mean precipitate radius $(\bar{R})$, the $\gamma^{\prime}$ volume fraction $\left(g^{\gamma}\right)$, and the average matrix concentrations $\left(\bar{c}_{i(k)}^{\gamma}\right)$ are calculated at each timestep:

$$
\begin{aligned}
& N=\sum_{k} n_{(k)} \\
& g^{\gamma^{\prime}}=\sum_{k} \frac{4 \pi}{3} R_{(k)}^{3} n_{(k)} \\
& \bar{R}=\frac{\sum_{k} R_{(k)} n_{(k)}}{\sum_{k} n_{(k)}} \\
& \bar{c}_{i(k)}^{\gamma}=\frac{c_{0, i}-\frac{4 \pi}{3} \sum_{k} n_{(k)} R_{(k)}^{3} c_{i(k)}^{\gamma^{* *}}}{1-g^{\gamma^{\prime}}}
\end{aligned}
$$

where $c_{0, i}$ is the nominal concentration of the alloy in element $i$.

In order to perform linked PFT-PSD simulations, the PSD model can be applied at different locations in the PFT simulation domain taking into account their concentrations, as shown in Figure 1.

\section{Results and discussion}

\section{PSD simulations on Ni-7.56at\%Al-8.56 at $\% \mathrm{Cr}$}

The PSD model was applied first to the isothermal heat treatment of $\mathrm{Ni}-7.56 \mathrm{at} \% \mathrm{Al}-8.56 \mathrm{at} \% \mathrm{Cr}$ for a total duration of $4 \times 10^{6} \mathrm{~s}$ at $600^{\circ} \mathrm{C}$. These conditions correspond to those employed by BoothMorrison et al. for the Atom Probe Tomography (APT) observations of $\gamma^{\prime}$ precipitates in Ni-Al-Cr alloys [19-21]. Initially, the alloy is assumed to be free of $\gamma^{\prime}$ precipitates. The diffusion matrix is an input of the model and is given in Table 1 . It was taken from the data published by Mao et al. [20], which is based on Monte-Carlo simulations for the diffusion-driven growth of $\gamma^{\prime}$ precipitates.
Table 1 : Diffusion coefficients $\left[\mathrm{m}^{2} \cdot \mathrm{s}^{-1}\right]$ in the $\gamma$ matrix used to simulate precipitation in Ni-7.56at\% Al-8.56at\% $\mathrm{Cr}$ at $600^{\circ} \mathrm{C}$ (obtained from [20]).

\begin{tabular}{|c|c|c|}
\hline & $\mathrm{Al}$ & $\mathrm{Cr}$ \\
\hline $\mathrm{Al}$ & $2.08 \times 10^{-20}$ & $8.59 \times 10^{-21}$ \\
\hline $\mathrm{Cr}$ & $8.13 \times 10^{-21}$ & $3.82 \times 10^{-21}$ \\
\hline
\end{tabular}

The other input parameters are summarised in Table 2. Nucleation is assumed to be homogeneous, with a potential nucleation sites density given by $N_{o}=N_{a v} / V_{m}$, where $N_{a v}$ is the Avogadro number and $V_{m}$ the constant molar volume $\left[\mathrm{m}^{3} \cdot \mathrm{mol}^{-1}\right]$.

Table 2 : Input parameters of the precipitation model applied to $\mathrm{Ni}-7.56 \mathrm{at} \% \mathrm{Al}-8.56 \mathrm{at} \% \mathrm{Cr}$ at $600^{\circ} \mathrm{C}$.

\begin{tabular}{|l|l|}
\hline$\sigma^{\gamma / \gamma^{\prime}}$ & $0.021 \mathrm{~J}^{-\mathrm{m}^{-2}}[19]$ \\
\hline$V_{m}$ & $6.8 \times 10^{-6} \mathrm{~m}^{3} \cdot \mathrm{mol}^{-1}$ \\
\hline$N_{o}$ & $8.9 \times 10^{28} \mathrm{~m}^{-3}$ \\
\hline
\end{tabular}

Figure 2 shows the evolution of (a) the mean radius $\bar{R}$, (b) the total number density $N$ and (c) the volume fraction of the $\gamma$ ' precipitates $g^{\gamma}$ calculated with the PSD model. During the initial stage (from 0 to $2000 \mathrm{~s}$ ), the phase transformation is mainly controlled by the nucleation of $\gamma$ 'precipitates in the supersaturated $\gamma$ matrix. This is shown by the quick increase of $N$ (Figure 2b).

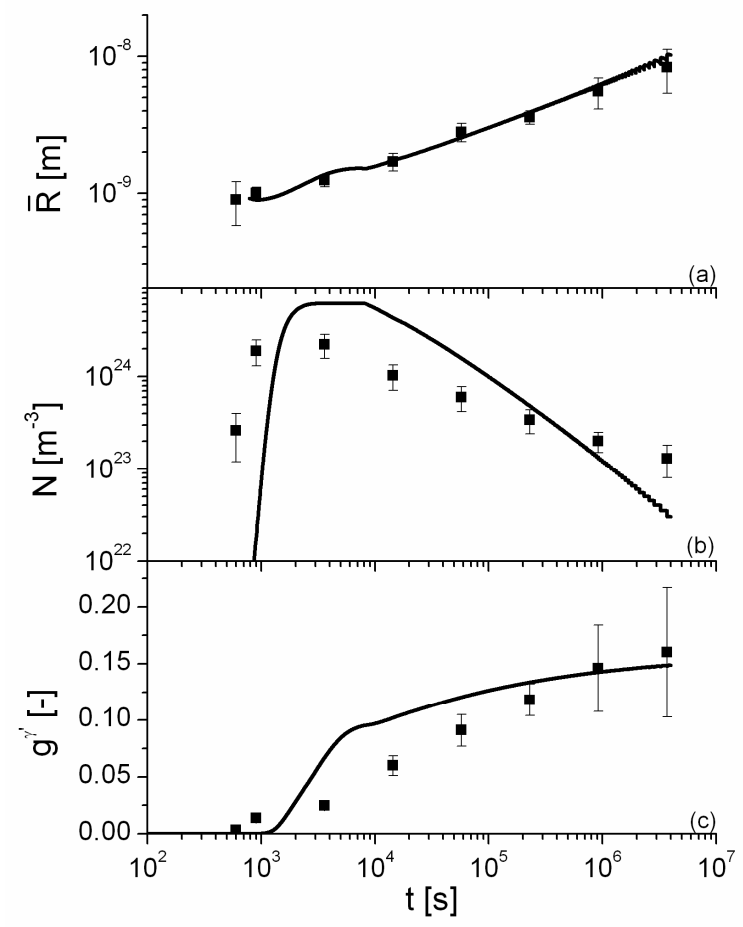

Figure 2 : Evolution of the mean precipitate radius (a), total number density $(b)$ and volume fraction $(c)$ of the $\gamma$ precipitates in $\mathrm{Ni}-7.56 \mathrm{at} \% \mathrm{Al}-8.56 \mathrm{at} \% \mathrm{Cr}$ at $600^{\circ} \mathrm{C}$. The black squares correspond to the experimental data of Booth-Morrison et al. [19], while the results obtained with the PSD model are represented with continuous lines. 
Growth can occur simultaneously to nucleation, which is characterized by a continuous increase of the precipitate mean radius and volume fraction, while $N$ keeps increasing. The increase of the $\gamma^{\prime}$ volume fraction reduces progressively the matrix supersaturation and thus the nucleation driving force. The precipitate density reaches a maximum value near $4 \times 10^{3} \mathrm{~s}$, which corresponds to the end of the nucleation process due to an insufficient nucleation driving force. During this initial growth regime, only diffusive growth and the Gibbs-Thomson effect control the evolution of the mean precipitate radius. The next regime $\left(4 \times 10^{3} \mathrm{~s}<t<10^{4} \mathrm{~s}\right)$ shows a decrease of the growth exponent. The total number density remains constant and the evolution of $g^{\gamma}$ is slowed down. Beyond $10^{4} \mathrm{~s}$, the calculation shows an increase of the slope of $\bar{R}(t)$ (logarithmic scale) and a decrease of the total precipitate density. The simultaneous increase of $\bar{R}$ and decay of $N$ indicates that some precipitates shrink and the smallest disappear. This process is controlled by the variations of the interface concentrations with the individual precipitate sizes through the Gibbs-Thomson effect. However, a pure coarsening regime would lead to time dependencies of $t^{1 / 3}$ and $t^{-1}$ for $\bar{R}(t)$ and $N(t)$, respectively. In simulations, such dependencies are only reached at a time larger than $10^{6} \mathrm{~s}$. Thus, the observed results correspond to a mixed growth and coarsening regime, with an increasing influence of coarsening as time increases.

Figure 3 shows the simulated size distributions, at $\mathrm{t}=1200$, 2000, $2800,10^{5}$ and $2 \times 10^{5} \mathrm{~s}$. These results were obtained from the precipitate number density in each class and the average size interval between adjacent classes. The maximum value of the distribution increases from 0 to $2000 \mathrm{~s}$. Simultaneously, the whole size distribution is shifted towards larger radii. This horizontal translation is more pronounced for the classes with higher radii, which also tends to broaden the distribution. As seen previously, this time interval $(0<t<2000 \mathrm{~s})$ corresponds to the concomitant nucleation and growth regime. From 2000 to 2800 s, a pure growth regime is reached and the horizontal displacement of the size distribution is more homogeneous. For ageing times of $10^{5} \mathrm{~s}$ and $2 \times 10^{5} \mathrm{~s}$ the size distributions are broadening and the peak value continuously decreases, this being associated with the decrease of the total precipitate density. This time interval characterizes a mixed growth and coarsening regime.

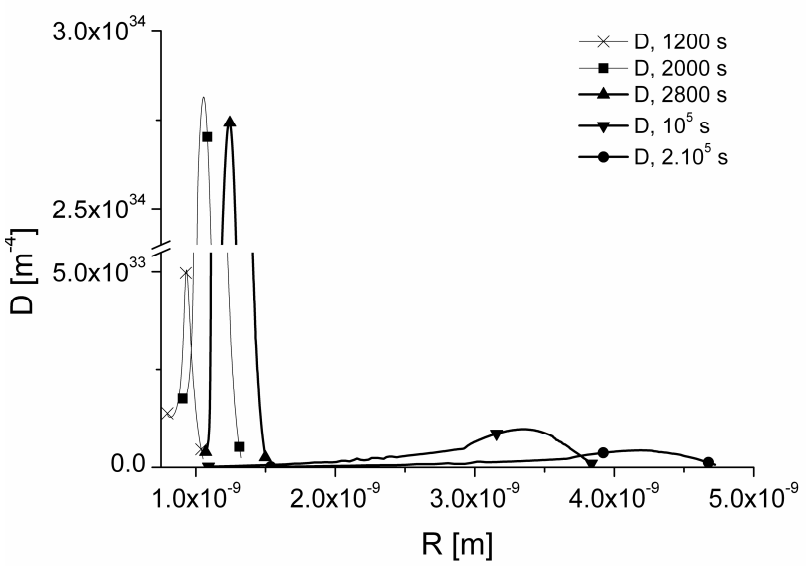

Figure 3 : Calculated size distributions at different heat treatment times.
In Figure 4 the paths of the interface concentrations have been represented in an isothermal section of the ternary phase diagram. As precipitation proceeds the matrix is depleted in $\mathrm{Al}$ and enriched in Cr. The final average matrix concentration calculated with the model lies approximately on the equilibrium tie-line (dotted line).

\section{Comparison with the experimental data}

The experimental data obtained by Booth-Morrison et al. [19] for the same alloy have been reported in Figure 2 and 4. As shown in Figure 2a, the evolution of the mean precipitate radius predicted with the PSD model is in very good agreement with the experimental data. The agreement is also satisfactory on Figure $2 \mathrm{~b}$, where the simulated and experimental values of $N$ are of the same order of magnitude. With respect to the experiments, nucleation occurs later, and the maximum of $N$ is higher in the simulation than in the experiment. The decrease of $N$ starts at $t=3600 \mathrm{~s}$ and $t=8000 \mathrm{~s}$ for measurements and simulations, respectively. The values of $d(\operatorname{Ln}(N)) / d(\operatorname{Ln}(t))$ differ significantly. In the simulations, the final value is close to -1 , which corresponds to the theoretical value predicted for the coarsening regime [19], whereas the slope is -0.42 for the last regime of the experimental data. As shown in Figure $2 b$ and $c$, the differences between the simulated and the measured values of $g^{\gamma}(t)$ are related to those observed for $N(t)$.

The simulated and measured precipitation paths shown in Fig. 4 both exhibit a progressive depletion of the matrix in $\mathrm{Al}$ and enrichment in Cr. The final average matrix concentration lies on the tie-line of the alloy only for the calculated path. One can note that the measured average matrix concentrations cross the solvus calculated for $R=1 \mathrm{~nm}$ and $R=2 \mathrm{~nm}$, and continue beyond the solvus calculated for an infinite precipitate radius. Important discrepancies between the calculated and measured interface concentrations are observed for the $\gamma^{\prime}$ phase. The initial Al concentrations differ substantially $(21$ at $\%$ for the APT observations, and 17 at $\%$ for the simulations), and the global depletion of $\mathrm{Cr}$ that is observed experimentally is not reproduced by the precipitation model.

In summary, the results of the simulation are globally in good agreement with the experimental data. However, some discrepancies are observed for the evolution of the total precipitate density, in particular during the nucleation and coarsening stages. The observed differences during the nucleation stage can be related to the nucleation law employed in the PSD model, which is formulated under restrictive assumptions for the diffusion kinetics and the nature of the nucleation sites. Only homogeneous nucleation is taken into account in the PSD model, which can be a severe limitation if the material contains dislocations or other defects enhancing the nucleation of $\gamma$ ' precipitates $[22,23]$. The implementation of a nucleation site density as an input parameter could improve the description of nucleation kinetics during the early stages of precipitation. To obtain better agreement with experimental results, a lower density of precipitates with a quicker nucleation would be needed, which could certainly be obtained with a heterogeneous nucleation law. Comparison with experimental results also shows that the increase of the $\gamma^{\prime}$ volume fraction during the growth stage is faster in the simulation than in the experiment. These differences can be related to the assumptions made for the growth of $\gamma^{\prime}$ precipitates in the $\gamma$ matrix. The mean field diffusion model does not take into 
account the local shape of the concentration profiles in the matrix, which can be strongly influenced by the relative position of the precipitates, especially in the case of systems featuring a high particles density [24, 25]. Furthermore, coarsening is assumed to occur only via diffusion fluxes in the matrix. Direct contacts between $\gamma^{\prime}$ precipitates leading to coalescence (or coagulation) events are not taken into account in the PSD approach. This can certainly influence the evolution of $N$. Finally, non-negligible differences of interface concentrations are observed in Figure 4. This point is directly linked to the thermodynamic database, which mainly influences the precipitation paths and the supersaturation calculations. As shown in Figure 4, the measured concentrations in the $\gamma$ and $\gamma^{\prime}$ phases do not lie on the calculated equilibrium tie-line, even for the longest ageing times. It is believed that a more accurate description of thermodynamic equilibria would improve the agreement between simulations and experiments.

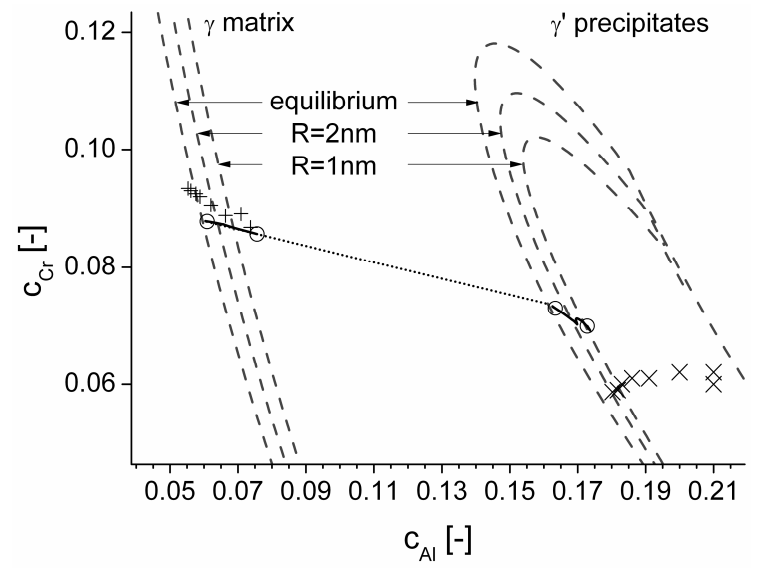

Figure 4 : Evolution of average concentrations in the $\gamma$ matrix and in the $\gamma$ precipitates during heat treatment of $\mathrm{Ni}-7.56 \mathrm{at} \% \mathrm{Al}$ $8.56 \mathrm{at} \% \mathrm{Cr}$ at $600^{\circ} \mathrm{C}$. The crosses correspond to the experimental data of Booth-Morrison et al. [19]. Simulation results are shown with thick black lines. The $\gamma$ and $\gamma$ solvus (dashed lines) were obtained from the dedicated thermodynamic database with $R=1$,

$2 \mathrm{~nm}$ and without accounting for the Gibbs-Thomson effect (equilibrium). The equilibrium tie-line is indicated by the dotted line.

\section{$\underline{\text { PFT-PSD simulations on } \mathrm{Ni}-19 \mathrm{at} \% \mathrm{Al}}$}

Another series of simulations was carried out by linking the PFT and PSD models to assess the influence of solidification and solution heat treatment on the formation of precipitates. This simulation was first performed for a binary $\mathrm{Ni}-19 \mathrm{at} \% \mathrm{Al}$ alloy and a DAS of $100 \mu \mathrm{m}$. Two points located at different depths in the primary phase: $x=0$ (core of the dendrite arm) and $x=66 \mu \mathrm{m}$ (periphery) were selected for PSD calculations. Table 3 and 4 summarize the main input parameters for the PFT and PSD calculations. The diffusion coefficients have been calculated using Thermo-Calc with the dedicated databases for Ni-based alloys. The temperature history used for the PFT calculation is shown in Figure 5. Heat extraction rates were imposed for PFT calculations. They are summarized in Table 3.

The evolution of the temperature and phase fractions is shown on Figure $5 \mathrm{a}$ and $\mathrm{b}$, respectively. Figure $6 \mathrm{a}$ and $\mathrm{b}$ shows the concentration profiles at different times during solidification and heat treatment.

Table 3 : Input parameters used for the PFT simulation of solidification and homogenization in Ni-19at\%Al.

\begin{tabular}{|c|c|}
\hline$c_{0, A l}$ & $19 \mathrm{at} \%$ \\
\hline$D_{A l}^{\gamma}$ & $1.23 \times 10^{-4} \cdot \mathrm{e}^{-248000 / \mathrm{RT}} \mathrm{m}^{2} . \mathrm{s}^{-1}$ \\
\hline$D_{A l}^{l}$ & $3 \times 10^{-9} \mathrm{~m}^{2} . \mathrm{s}^{-1}$ \\
\hline$D A S$ & $10^{-4} \mathrm{~m}$ \\
\hline \multirow{3}{*}{$\dot{H}\left[\mathrm{~W} . \mathrm{m}^{-3}\right]$} & $-2.10^{6}$ from 0 to $4150 \mathrm{~s}$ \\
\cline { 2 - 2 } & 0 from 4150 to $4200 \mathrm{~s}$ \\
\cline { 2 - 2 } & $2.10^{6}$ from 4200 to $6864 \mathrm{~s}$ \\
\cline { 2 - 2 } & 0 from 6864 to $7800 \mathrm{~s}$ \\
\hline
\end{tabular}

Table 4 : Input parameters used for the PSD simulations in the PFT domain. $c_{A l}^{\gamma, e q}$ and $c_{A l}^{\gamma^{\prime}, e q}$ are the equilibrium concentrations in the rand $\gamma$ phase.

\begin{tabular}{|c|c|}
\hline$c_{0, A l}$ & $\begin{array}{c}18.5 \mathrm{at} \% \text { at } \mathrm{x}=0 \mu \mathrm{m}(\mathrm{A}) \\
19.2 \mathrm{at} \% \text { at } \mathrm{x}=66 \mu \mathrm{m}(\mathrm{B})\end{array}$ \\
\hline$c_{A l}^{\gamma, e q}$ & $14.38 \mathrm{at} \%$ \\
\hline$c_{A l}^{\gamma, e q}$ & $23.08 \mathrm{at} \%$ \\
\hline$\Omega$ & 0.473 and 0.554 \\
\hline$\sigma^{\gamma / \gamma^{\prime}}$ & $0.021 \mathrm{~J} \cdot \mathrm{m}^{-2}$ \\
\hline$V_{m}$ & $6.8 \times 10^{-6} \mathrm{~m}^{3} \cdot \mathrm{mol}^{-1}$ \\
\hline$N_{o}$ & $8.9 \times 10^{28} \mathrm{~m}^{-3}$ \\
\hline$D_{A l}^{l}$ & $9.0 \times 10^{-16} \mathrm{~m}^{2} \cdot \mathrm{s}^{-1}$ \\
\hline
\end{tabular}

At $t=1000 \mathrm{~s}$ the domain is composed of primary $\gamma$ and liquid. The $\mathrm{Al}$ concentration in the liquid increases progressively due to partitioning and the solid/liquid interface moves towards the right of the domain. Near the end of solidification, the $\gamma$ ' phase appears in the interdendritic region, and grows at the expense of the liquid. This result does not correspond to the phase equilibrium given by the equilibrium diagram, which indicates a $\gamma$ solid solution in Ni$19 \mathrm{at} \% \mathrm{Al}$ when the last liquid disappears. This result can naturally be explained by the finite diffusion kinetics, namely in the solid, which leads to a microsegregation profile that is closer to the Scheil approximation than the Lever rule.

When the temperature is below the solvus $(t=6000 \mathrm{~s}), \gamma^{\prime}$ grows due to an incoming flux of $\mathrm{Al}$ in the interdendritic region. This leads to an $\mathrm{Al}$ concentration profile exhibiting a maximum in the vicinity of the $\gamma / \gamma^{\prime}$ interface. During the solution/homogenization sequence a positive concentration gradient is again obtained in primary $\gamma$ due to the increase of the equilibrium concentration at the $\gamma / \gamma^{\prime}$ interface $(t=6850 \mathrm{~s})$. As a consequence, $\gamma^{\prime}$ dissolves progressively before fully disappearing. The last stage of the heat treatment $(t=7800 \mathrm{~s})$ corresponds to a damping process of the concentration profiles. A and B indicate the points located at $x=0$ and $x=66 \mu \mathrm{m}$, which corresponds to the conditions used as an input for the PSD calculations.

Figure $7 \mathrm{a}, \mathrm{b}$, and $\mathrm{c}$ shows the simulated evolutions of the average precipitates radius, density and phase fraction, respectively. Ni18.5at\% $\mathrm{Al}$ and $\mathrm{Ni}-19.2 \mathrm{at} \% \mathrm{Al}$ refer to the compositions obtained from the PFT calculation at $t=7800 \mathrm{~s}$ at positions $\mathrm{A}$ and $\mathrm{B}$, 
respectively. The maximum volume fraction of $\gamma^{\prime}$ reached in the simulation is higher for alloy B than for alloy A, due to the higher $\mathrm{Al}$ content. For these two alloys, the overall precipitation kinetics differ mainly during the nucleation stage, as can be seen in Figure 7. Due to the lower supersaturation in alloy A, the driving force for nucleation is smaller than for alloy B (see Table 5), which delays the formation of $\gamma^{\prime}$ precipitates. The maximum precipitate densities are 5.4 and $7.0 \times 10^{25} \mathrm{~m}^{-3}$ for alloys $\mathrm{A}$ and $\mathrm{B}$, respectively. For both alloys, the growth/coarsening regime starts at about $\mathrm{t}=10^{-2} \mathrm{~s}$ and the evolutions of $\bar{R}(t)$ and $N(t)$ are then almost identical. The slopes $d(\operatorname{Ln}(\bar{R}) / d(\operatorname{Ln}(t)) \quad$ and $\mathrm{d}(\operatorname{Ln}(N)) / \mathrm{d}(\operatorname{Ln}(t))$ calculated for $t>1 \mathrm{~s}$ are 0.32 and -0.97 , respectively, which is close to the exponents predicted by the LSW theory [26, 27]. The LSW theory applies if the radius of the precipitates is controlled only by coarsening. In the framework of this theory, the growth rate mainly depends on $D, \sigma^{\gamma \gamma}$ and $V_{m}$. As these values are the same for the two alloys, the evolutions of the mean precipitate radius are similar for the two nominal concentrations.

The precipitation behaviour calculated for locations A and B in the dendrite arm are quite similar in this example. However, larger influences are expected in alloys containing more solute elements and/or higher concentrations. Heavy elements, which diffuse much slower than $\mathrm{Al}$, are likely to exhibit more pronounced gradients at the end of the solution heat treatment. Such effects can be investigated with this approach.

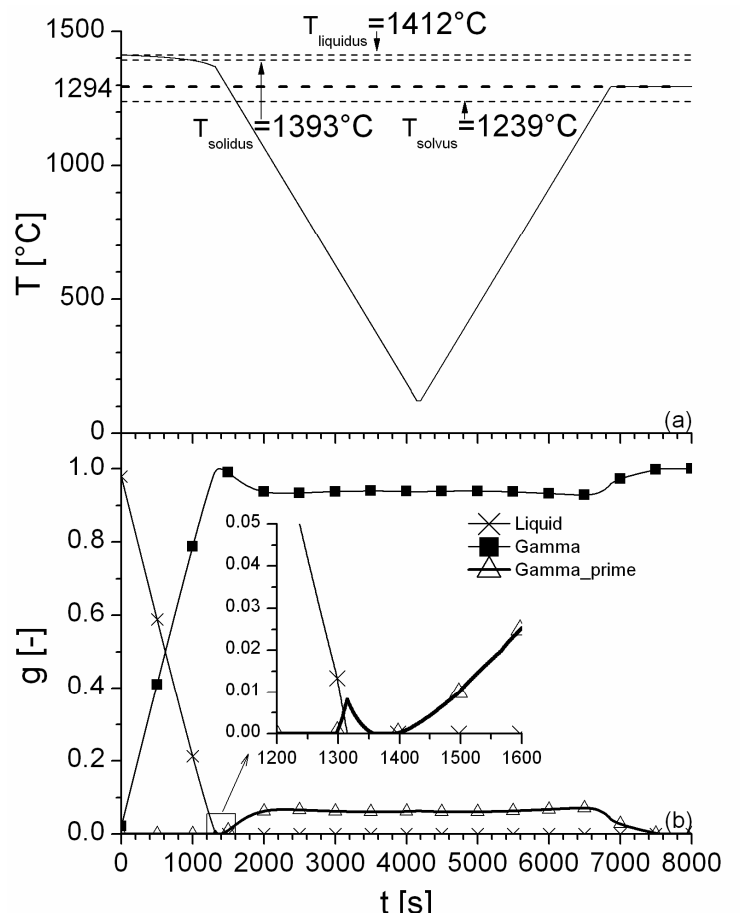

Figure 5 : Temperature history (a) and phase fractions (b) during solidification and solution heat treatment in Ni-19at\%Al. The liquidus, solidus and solvus temperatures are indicated by dashed lines on the temperature history (a).

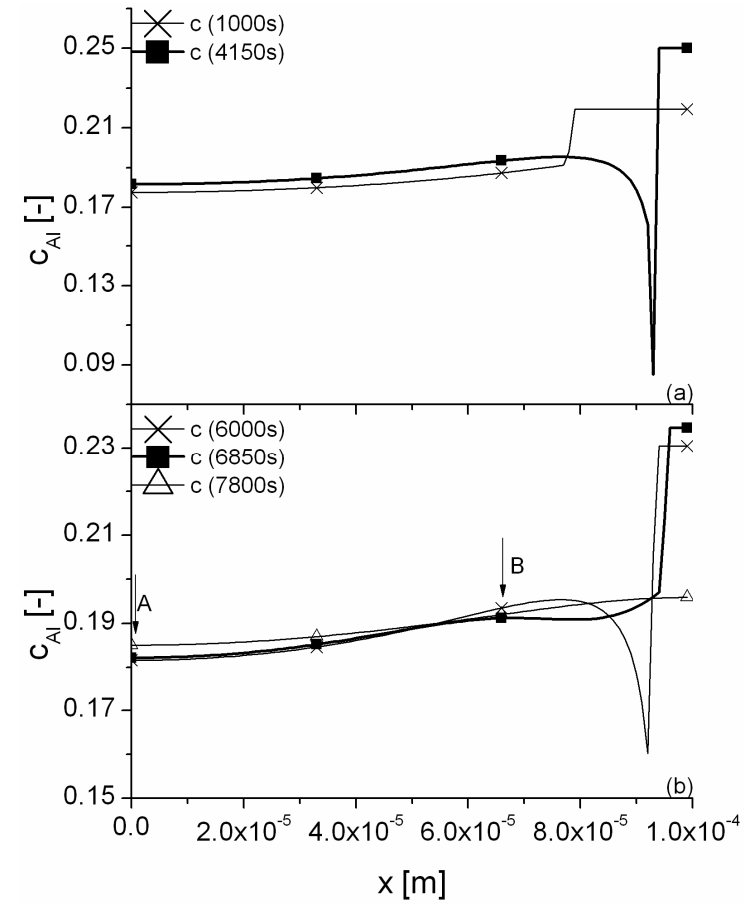

Figure 6 : Concentration profiles calculated with the PFT model during solidification and heat treatments. $A$ and $B$ points correspond to $x=0$ and $x=66 \mu \mathrm{m}$, respectively.

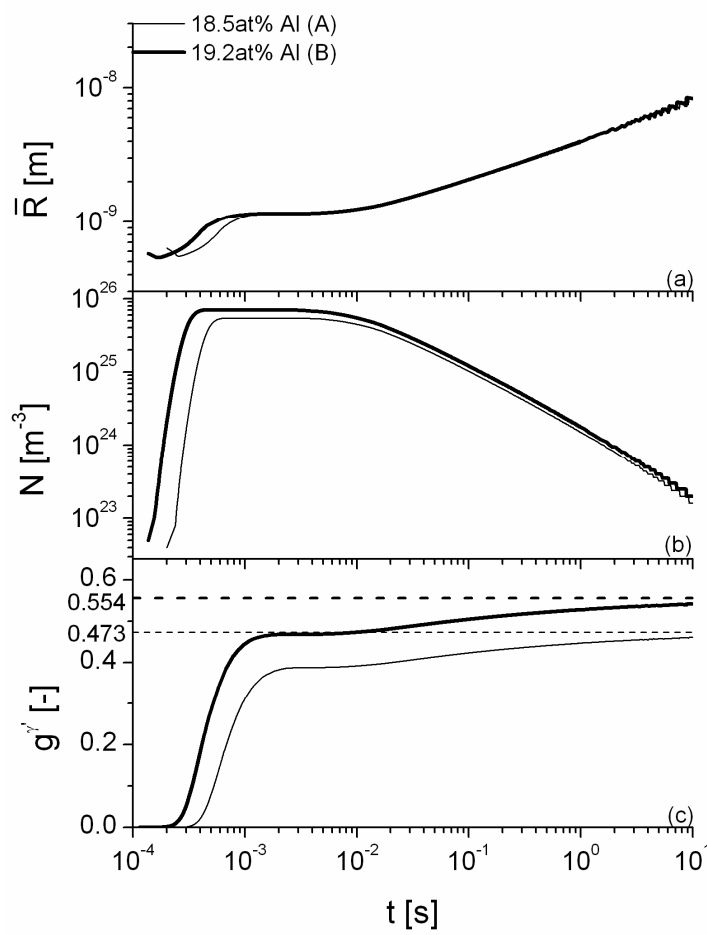

Figure 7 : Evolution of the mean radius (a), total number density (b) and volume fraction (c) of the $\gamma$ precipitates for the $\mathrm{Ni}$ $18.5 \mathrm{at} \% \mathrm{Al}$ and $\mathrm{Ni}-19.2 \mathrm{at} \% \mathrm{Al}$ alloys heat treated at $900^{\circ} \mathrm{C}$. 
Table 5 : Initial nucleation driving forces for $\mathrm{Ni}-18.5 \mathrm{at} \% \mathrm{Al}$ and

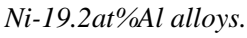

\begin{tabular}{|c|c|c|}
\hline$c_{0, A l}$ & $18.5 \mathrm{at} \%$ & $19.2 \mathrm{at} \%$ \\
\hline$\Delta G_{v}$ & $1.8 .10^{8} \mathrm{~J} . \mathrm{m}^{-3}$ & $2.1 .10^{8} \mathrm{~J}^{-3} \mathrm{~m}^{-3}$ \\
\hline
\end{tabular}

\section{Conclusions}

The simulations performed with the PSD precipitation model on a ternary $\mathrm{Ni}-\mathrm{Al}-\mathrm{Cr}$ model alloy are in satisfactory agreement with the experimental results of Booth-Morrison et al. [19]. The observed discrepancies can be explained by the assumption of homogeneous nucleation which is exempt of any adjustable parameter and does not allow to account for the role of nucleation sites such as dislocations or vacancies. Considering also that the thermodynamic and mobility [20] data were used without any adjustment, it can be concluded that the model captures the physics of precipitation of this system in a very satisfactory manner.

The effect of residual microsegregation has been addressed by performing precipitation simulations using concentrations at different locations in a dendrite arm in a binary $\mathrm{Ni}-19 \mathrm{at} \% \mathrm{Al}$ alloy. Relative concentration differences of only a few percents can already cause non-negligible changes of precipitates densities and volume fractions. In industrial multicomponent superalloys, the residual segregation can be much more pronounced due to slow diffusing elements, complex partitioning and uphill diffusion. A full coupling such as presented in [11] will be considered in the future to account for the reciprocal influence of precipitation and solute diffusion on the scale of the dendrite arms for multicomponent industrial alloys.

\section{Acknowledgements}

This work is supported by Snecma-SAFRAN group. The authors gratefully acknowledge Prof. M. Rappaz for interesting discussions on the subject of this work.

\section{References}

1. J. H. Weber, "Nickel-based Superalloys: Alloying", in: K. H. J. Buschow, W. C. Robert, C. F. Merton, I. Bernard, J. K. Edward, M. Subhash, V. Patrick (Eds.), Encyclopedia of Materials. Science and Technology, Elsevier, Oxford, 2001, 6146-6149.

2. J. S. Langer, "Kinetics of nucleation in near-critical fluids", Physical review. A, Atomic, molecular, and optical physics, 21 (1980), 948-958.

3. R. Kampmann, R. Wagner, "Kinetics of precipitation in metastable binary alloys - theory and application to $\mathrm{Cu}-1.9$ at $\% \mathrm{Ti}$ and Ni-14at\% Al", in: P. Haasen, V. Gerold, R. Wagner, F. Ashby Michael (Eds.), Decomposition of Alloys: The Early Stages, Pergamon Press, Sonnenberg, W Ger, 1984, pp. 91-103.

4. J. D. Robson, P. B. Prangnell, "Dispersoid precipitation and process modelling in zirconium containing commercial aluminium alloys", Acta Materialia, 49 (2001), 599-613.

5. M. Nicolas, A. Deschamps, "Characterisation and modelling of precipitate evolution in an Al-Zn-Mg alloy during non-isothermal heat treatments", Acta Materialia, 51 (2003), 6077-6094.

6. M. Serriere, C. A. Gandin, E. Gautier, P. Archambault, M. Dehmas, "Modeling of precipitation coupled with thermodynamic calculations", Materials Science Forum, 396-402 (2002), 747752 .

7. Q. Chen, J. Jeppsson, J. Agren, "Analytical treatment of diffusion during precipitate growth in multicomponent systems", Acta Materialia, 56 (2008), 1890-1896.

8. C. A. Gandin, Y. Bréchet, M. Rappaz, G. Canova, M. Ashby, H. Shercliff, "Modelling of solidification and heat treatment for the prediction of yield stress of cast alloys", Acta Materialia, 50 (2002), 901-927.

9. A. Håkonsen, D. Mortensen, S. Benum, T. Pettersen, T. Furu, "Modelling the metallurgical reactions during homogenisation of an AA3103 alloy", in: R. Peterson (Ed.) Light Metals: Proceedings of Sessions, TMS Annual Meeting (Warrendale, Pennsylvania), Seattle, WA, 2002, pp. 793-800.

10. A.-L. Dons, "The Alstruc homogenization model for industrial aluminum alloys", Journal of Light Metals, 1 (2001), 133-149.

11. C. A. Gandin, A. Jacot, "Modeling of precipitate-free zone formed upon homogenization in a multi-component alloy", Acta Materialia, 55 (2007), 2539-2553.

12. A. Jacot, M. Rappaz, "A pseudo-front tracking technique for the modelling of solidification microstructures in multicomponent alloys", Acta Materialia, 50 (2002), 1909-1926.

13. Q. Du, A. Jacot, "A two-dimensional microsegregation model for the description of microstructure formation during solidification in multicomponent alloys: Formulation and behaviour of the model", Acta Materialia, 53 (2005), 3479-3493.

14. K. C. Russel, "Nucleation in solids: the induction and steady state effects", Advances in Colloid and Interface Science, 13 (1980), 215-318.

15. H. I. Aaronson, J. K. Lee, "The kinetic equations of solid-solid nucleation theory", in: N. Y. A. I. o. M. New York, Metallurgical and Petroleum Engineers (Ed.) Lectures on the theory of phase transformations, 1982.

16. M. Perez, M. Dumont, D. Acevedo-Reyes, "Implementation of classical nucleation and growth theories for precipitation", Acta Materialia, 56 (2008), 2119-2132.

17. P. Maugis, M. Gouné, "Kinetics of vanadium carbonitride precipitation in steel: A computer model", Acta Materialia, 53 (2005), 3359-3367

18. H. Aaron, G. Kotler, "Diffusion-Limited Phase Transformations: A Comparison and Critical Evaluation of the Mathematical Approximations", Journal of Applied Physics, 41 (1970), 4404

19. C. Booth-Morrison, J. Weninger, C. K. Sudbrack, Z. Mao, R. D. Noebe, D. N. Seidman, "Effects of solute concentrations on kinetic pathways in Ni-Al-Cr alloys", Acta Materialia, 56 (2008), 3422-3438.

20. Z. Mao, C. Booth-Morrison, C. K. Sudbrack, G. Martin, D. N. Seidman, "Kinetic pathways for phase separation: An atomicscale study in Ni-Al-Cr alloys", Acta Materialia, (2011).

21. C. K. Sudbrack, K. E. Yoon, R. D. Noebe, D. N. Seidman, "Temporal evolution of the nanostructure and phase compositions in a model Ni-Al-Cr alloy", Acta Materialia, 54 (2006), 31993210.

22. A. J. Ardell, R. B. Nicholson, "On the modulated structure of aged Ni-Al alloys", Acta Metallurgica, 14 (1966), 1295-1309.

23. T. Grosdidier, A. Hazotte, A. Simon, "Precipitation and dissolution processes in $\gamma / \gamma^{\prime}$ single crystal nickel-based superalloys", Materials Science and Engineering A, 256 (1998), 183-196.

24. P. W. Voorhees, M. E. Glicksman, "Solution to the multiparticle diffusion problem with applications to Ostwald ripening-I. Theory", Acta Metallurgica, 32 (1984), 2001-2011. 
25. P. W. Voorhees, M. E. Glicksman, "Solution to the multiparticle diffusion problem with applications to ostwald ripening-II. Computer simulations", Acta Metallurgica, 32 (1984), 20132030.

26. I. M. Lifshitz, V. V. Slyozov, "The kinetics of precipitation from supersaturated solid solutions", Journal of Physics and Chemistry of Solids, 19 (1961), 35-50.

27. C. Wagner, "Theorie der Alterung von Niederschlägen durch Umlösen (Ostwald-Reifung)", Zeitschrift für Elektrochemie, 65 (1961), 581-591. 